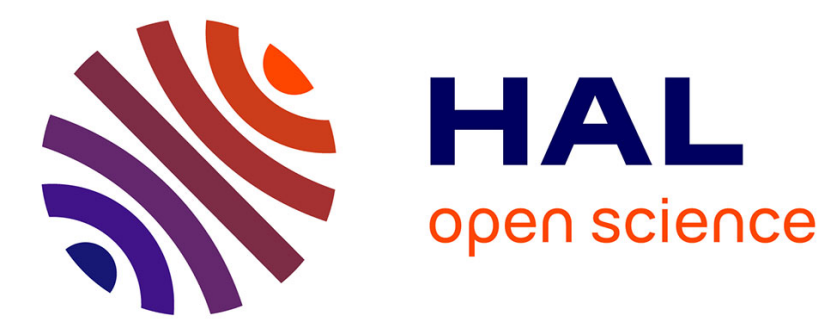

\title{
Troubles de la marche et chutes chez le sujet âgé
}

Didier Deffond

\section{To cite this version:}

Didier Deffond. Troubles de la marche et chutes chez le sujet âgé. Revue du Rhumatisme monographies, 2019, 86, pp.178 - 182. 10.1016/j.monrhu.2019.02.005 . hal-03484744

\section{HAL Id: hal-03484744 \\ https://hal.science/hal-03484744}

Submitted on 20 Dec 2021

HAL is a multi-disciplinary open access archive for the deposit and dissemination of scientific research documents, whether they are published or not. The documents may come from teaching and research institutions in France or abroad, or from public or private research centers.
L'archive ouverte pluridisciplinaire HAL, est destinée au dépôt et à la diffusion de documents scientifiques de niveau recherche, publiés ou non, émanant des établissements d'enseignement et de recherche français ou étrangers, des laboratoires publics ou privés.

\section{다)(1) $\$$}

Distributed under a Creative Commons Attribution - NonCommercial| 4.0 International 


\section{Troubles de la marche et chutes chez le sujet âgé Gait disorders and falls in the elderly}

Didier Deffond $(1,2)$

1 Service de neurologie et centre recherche ressources mémoire. CHRU de Clermont-ferrand, hôpital Gabriel Montpied. BP 69, 63003 Clermont-ferrand cedex 1

2 Pôle de gérontologie. Hôpital Guy Thomas. BP 167, 63204 Riom cedex

Correspondance : Dr Didier Deffond. CMRR hôpital Gabriel Montpied. BP 6963003 Clermont-ferrand cedex 1.

Tel : $0473751151 ;$ fax : 0473754411,

adresse e-mail : ddeffond@chu-clermontferrand.fr 


\section{Résumé}

Les troubles de la marche et les chutes chez les sujets âgés deviennent un problème majeur de santé publique de par leur fréquence et leurs conséquences en termes de perte d'autonomie et de coûts de prise en charge. Les altérations du système locomoteur avec le vieillissement, ainsi que la fréquence des pathologies affectant directement ou indirectement ce système, expliquent l'incidence très élevée de ces troubles qui croit avec l'avancée en âge. Les interventions visant à réduire le risque de récidive de chute ou à améliorer une locomotion déjà perturbée ont des résultats décevants. Un programme de prévention primaire pourrait être plus efficace, mais reste à tester dans une large population présymptomatique.

Mots clefs : vieillissement, marche, équilibre, chute, prévention.

\section{Summary}

Gait disorders and falls in elderly persons become a major public health concern because of high occurrence and consequences on functional independence and costs of care. Damages caused by growing age to the locomotor system together with numerous pathologies affecting directly or indirectly gait and balance, explain high incidence of such troubles in advanced age. Mutifactorial intervention aimed to prevent subsequent fall or to improve gait when already impaired give poor results. A primary prevention program should be more effective but remains to be studied in large presymptomatic population.

Key words : ageing, gait, balance, fall, prevention. 
Dans la pratique médicale quotidienne, la silhouette chancelante du sujet âgé chuteur est devenue familière. Et pour cause : 30 \% des plus de 65 ans chutent au moins une fois par an, proportion qui atteint $50 \%$ chez les plus de 80 ans [1,2] et environ $15 \%$ chutent plusieurs fois par an. La fréquence de ce phénomène est attribuable d'une part au vieillissement " physiologique » du système d'équilibration et d'autre part à la grande fréquence de diverses pathologies affectant le système locomoteur dans cette tranche de la population.

\section{Tenir debout et marcher : un défi}

Élever son centre de masse à un mètre du sol et le contenir dans un polygone de sustentation de quelques centimètres carrés, tout en étant capable d'induire une succession de déséquilibres pour de déplacer en marchant, fait appel à des mécanismes extrêmement complexes et fragiles que la robotique échoue toujours à reproduire et même à approcher.

Le système comprend des afférences sensitives et sensorielles de trois ordres : visuelles, vestibulaires et proprioceptives.

La vision permet de situer l'individu dans l'espace et d'orienter les déplacements vers un but.

Le système vestibulaire renseigne sur la position de la tête et du cou et sur les accélérations linéaires et angulaires. Il est connecté : 1- avec les voies oculomotrices, le réflexe vestibulo-oculaire permet de stabiliser le regard lors des mouvements, 2- au cervelet qui module et coordonne l'activité musculaire et 3- avec la moelle par le faisceau vestibulospinal qui permet de moduler l'activité musculaire cervicale [3].

Les afférences proprioceptives conscientes et inconscientes relayent respectivement par le thalamus et le cervelet et transmettent les informations sur la position des segments de membres, le degré d'étirement des différents groupes musculaires, les pressions exercées sur la plante des pieds et les articulations.

Les informations provenant de ses trois sources convergent vers le cortex prémoteur, point de départ des efférences motrices qui relayent par les noyaux gris où les informations sont fusionnées pour générer réflexes de posture et automatisme de marche. Le cervelet placé en dérivation sur les voies efférentes corrige « en temps réel » toute déviation du schéma moteur initial et ajuste tonus musculaire et précision [3]. 
L'ensemble du système de posture et d'équilibration fonctionne comme un tout, ses éléments sont interconnectés et agissent en coordination. Dans certaines limites, si une des composantes dysfonctionne, une compensation par les autres acteurs du système peut permettre une compensation et une locomotion sinon normale du moins adaptée. Avec l'avancée en âge toutes les composantes du système voient leur efficacité réduite.

\section{Le vieillissement et ses conséquences sur la posture et la}

\section{marche}

Lorsque l'on tente d'étudier les conséquences du vieillissement sur l'organisme, deux questions se posent invariablement : 1) comment définir un sujet « âgé » ? Et 2) qu'est-ce que le vieillissement " normal » ? Si la réponse à la première question peut être assez simple en fixant une borne d'âge (en général 65 ans), la réponse à la deuxième question est beaucoup plus difficile. En effet de nombreuses pathologies sont associées au vieillissement et peuvent affecter la marche et l'équilibre (arthropathies, pathologies neurodégénératives et cérébro-vasculaires, affections de l'œil et de l'oreille interne...) un sujet âgé indemne de toutes ces pathologies est-il « normal » ou bien représente-t-il l'exception ? Cependant, il n'en reste pas moins que le vieillissement en lui-même affecte les performances globales des individus [4].

La perception visuelle décroît dès 50 ans, qu'il s'agisse de l'acuité centrale ou périphérique, de la perception des contrastes et de la profondeur [5].

L'efficacité du système vestibulaire s'émousse progressivement en lien avec des altérations et une réduction du nombre des cellules ciliées et des fibres myélinisées du nerf vestibulaire [6]. Cette " presbyvestibulie » qui ne s'accompagne pas nécessairement de vertiges car l'atteinte est souvent symétrique, va de pair avec la presbyacousie liée à l'âge, et de fait, la déficience auditive est un facteur de risque de chute chez le sujet âgé [7].

La somesthésie devient moins précise du fait d'une raréfaction et d'une diminution de la sensibilité des baro et mécanorécepteurs périphériques. Les vitesses de conduction nerveuse diminuent, ralentissant le transfert d'information vers le système nerveux central [8].

Les organes effecteurs deviennent aussi moins efficaces : la masse musculaire est réduite de $30 \%$ chez des sujets de 80 ans par rapport à des adultes jeunes, force et puissance décroissent avec l'âge 
de 3,5\% par an pour cette dernière au-delà de 65 ans en lien avec la raréfaction des fibres de type II [9]. À cela s'ajoute une réduction de l'élasticité des tissus tendineux et ligamentaires. Ces changements impliquent, entre autres, des réactions moins efficaces face à une situation de déséquilibre.

Il faut aussi prendre en compte les conséquences du vieillissement cognitif « normal ». Si la marche est un processus largement automatisé, elle reste sous la supervision du cortex préfrontal et donc des capacités exécutives. Or, aussi bien anatomiquement que fonctionnellement, c'est ce système qui subit les plus grandes altérations avec l'âge : rapidité de traitement des informations, flexibilité mentale, capacité d'anticipation déclinent avec le vieillissement $[10,11]$. Une des conséquences est une plus grande difficulté à effectuer des doubles tâches. Chez le sujet âgé, l'efficacité moindre du système d'équilibration implique de consacrer plus de ressources attentionnelles aux déplacements et donc à avoir moins de disponibilités cognitives pour effectuer une tâche concomitante. L'illustration est donnée par les épreuves de type " talking while walking ", plus la marche ralentie en situation conversationnelle et a fortiori si elle est impossible, plus le risque de chute est élevé [12].

\section{Les conséquences fonctionnelles}

\subsection{Adaptation appropriee}

La réduction globale des performances sensorimotrices entraîne des modifications de la marche et de la posture y compris chez des sujets en état de vieillesse « optimale » [4] : ralentissement de la vitesse de marche, réduction de l'amplitude des pas, léger élargissement de la base, attitude en légère antéflexion. Un sujet jeune qui doit traverser une patinoire s'adapte de la même manière à l'environnement ; pour un sujet âgé ces adaptations constituent une réponse appropriée à la réduction des capacités intrinsèques de l'individu et avec Nutt et al [13] on peut considérer que cette marche précautionneuse (« cautious gait ») est normale chez le sujet âgé, et non associée à un risque accru de chutes [14]. Elle s'accentue avec les âges extrêmes [4].

\subsection{Marche pathologique}

Chez un certain nombre de sujets cette marche précautionneuse va évoluer vers une marche pathologique (voire une impossibilité de verticalisation) qui entraîne un handicap fonctionnel retentissant sur les activités de la vie quotidienne. Cette détérioration de la marche peut survenir après une première chute d'où la dénomination de syndrome post-chute [15], qui désigne l'apparition, dans les suites immédiates de la chute, de difficultés de marche sévères voire d'une impossibilité totale avec un pronostic fonctionnel et vital très péjoratif [15]. II faut noter toutefois que dans 30 à $50 \%$ des cas 
ces difficultés apparaissent spontanément et progressivement $[16,17]$. Une certaine confusion vient de l'expression de « syndrome de peur de la chute » d'origine anglo-saxonne (« fear of falling » ou FOF) qui recouvre des réalités fort différentes depuis une marche très détériorée jusqu'à la simple réponse affirmative à la question : avez-vous peur de tomber ? De ce fait la prévalence est très diversement appréciée de 3 \% jusqu'à 85 \% chez les sujets les plus âgés [16]... C'est pourquoi les termes de syndrome de désadaptation psychomotrice (SDPM) ou de stasobasophobie proposé par Garcin (cité par Nutt et al [13]) semblent plus adaptés, et désignent une altération sévère des possibilités de marche en l'absence de pathologie clairement identifiée. Le spectre clinique est large d'une marche en rétropulsion, à très petits pas magnétiques avec recherche d'appui sur les meubles, les murs ou une tierce personne jusqu'à l'astasie abasie complète [13]. Lorsque la marche est possible, une réassurance même minime suffit souvent à améliorer les possibilités fonctionnelles.

Les déterminants de cette détérioration de la marche sont multiples à la fois physiques et psychologiques, une grande anxiété vis-à-vis de l'activité locomotrice fait partie du tableau, des éléments dépressifs sont fréquents et constituent un facteur aggravant [16]. Les troubles cognitifs jouent aussi un rôle important, même en l'absence de démence caractérisée, devant un SDPM, le risque d'évolution démentielle à 5 ans est 3 à 5 fois supérieur à la population générale [18].

Les principaux facteurs de risque du SDPM sont : un premier épisode de chute (le syndrome postchute [15]), le sexe féminin, la prévalence augmente aussi avec l'âge, particulièrement chez les 85 et plus [16].

Les conséquences sont : un risque de chute accru, une perte d'autonomie avec réduction des activités et retrait social, un taux d'institutionnalisation et de décès à court terme très élevé [15].

\subsection{Les chutes}

Nous considérerons ici les chutes sans malaise ni perte de connaissance qui seront évoquées au chapitre suivant.

Comme nous l'avons vu, marche pathologique et chutes ne sont pas nécessairement associées chez un individu donné, mais l'une est facteur de risque de l'autre et réciproquement. Ainsi le syndrome de désadaptation psychomotrice est un des principaux facteurs de risque de chute, au même titre qu'un premier épisode ou que l'avancée en âge ou encore une quelconque pathologie aiguë ou chronique $[2,19]$. La consommation de psychotropes multiplie par 2 à 3 le risque de chute et de fracture $[2,20,21]$. Enfin le déclin cognitif et a fortiori un état démentiel sont un autre déterminant très puissant et pas toujours bien évalué [18]. Ces derniers facteurs en altérant le discernement empêchent la 
prise en compte appropriée des conditions environnementales mais aussi des propres capacités de l'individu.

Les conséquences des chutes peuvent être dramatiques dans cette tranche d'âge ; en France en 2004, près de 14000 décès chez les plus de 65 ans sont en rapport direct avec une chute, avec des taux de mortalité de 71.6/100000 chez les 75-84 ans et de $442 / 100000$ chez les plus de 85 ans, 450000 personnes âgées ont été admises aux urgences pour chute, $37 \%$ hospitalisées ( $44 \%$ chez les plus de 90 ans) [22]. 10 à $15 \%$ des chutes entraînent une hospitalisation et environ $5 \%$ des lésions traumatiques graves, principalement des fractures qui touchent majoritairement l'avant-bras et le poignet chez les sujets $<75$ ans alors que les fractures de hanche sont plus fréquentes chez les plus âgés ; probablement du fait d'un ralentissement de la mise en œuvre des réflexes de protection [22]. Les traumatismes crâniens sont plus rares (13 \% des admissions) mais entraînent une importante morbi-mortalité. Les chutes traumatiques sont un facteur de risque indépendant d'institutionnalisation avec un taux de $38 \%$, et de décès dans l'année suivante $[1,23]$. En dehors des traumatismes physiques, il est important de prendre en compte l'impact psychologique, souvent majeur, conduisant au syndrome post-chute avec régression psycho motrice qui peut aboutir à la grabatisation [15].

Les dépenses engendrées par les chutes et leurs conséquences chez les séniors sont considérables, aux États-Unis, les coûts médicaux directs estimés sont de l'ordre de 30 milliards de dollars annuels [24].

\section{L'examen du patient chuteur}

Toute chute doit être considérée comme un événement sérieux et requiert un examen clinique complet et rigoureux ainsi que le recours à d'éventuels examens complémentaires selon le contexte. L'interrogatoire doit d'abord évaluer les circonstances de la chute : lors de la marche ou d'un transfert, l'intervention d'un obstacle extérieur. Bien entendu il faut s'enquérir de l'éventualité d'un malaise ou d'une perte de connaissance. L'immédiat post-chute doit être questionné et notamment si le sujet a pu se relever seul et sinon combien de temps est-il resté au sol, y a-t-il eu confusion dans les suites ?q

L'examen physique doit avoir pour objectif prioritaire d'évaluer les conséquences traumatiques de la chute et le cas échéant d'orienter la prise en charge vers une structure adaptée. En second lieu, il doit permettre la mise en évidence d'une éventuelle pathologie spécifique, l'examen clinique recher- 
chera un déficit sensitif ou moteur, un syndrome parkinsonien, une arthropathie des membres inférieurs, une déficience visuelle ou auditivo-vestibulaire, une évaluation cognitive minimum doit faire partie de l'examen standard. La recherche d'une hypotension orthostatique (HTO) doit être systématique (après 5 minutes de clinostatisme, prise de pression artérielle, puis après 1 et 3 minutes d'orthostatisme ; une HTO se définit par une baisse de $20 \mathrm{mmHg}$ de la systolique et/ou de $10 \mathrm{mmHg}$ de la diastolique, ou respectivement 30 et $15 \mathrm{mmHg}$, si la systolique en position couchée est $\geq$ à 150 $\mathrm{mmHg}$ ) ; et est très fréquemment retrouvée dans cette population (jusqu'à $30 \%$ ), même s'il n'y a pas d'arguments à l'interrogatoire [25]. L'examen clinique sera complété par un électrocardiogramme en cas de malaise ou perte de connaissance.

L'interprétation de l'examen doit être pondérée en fonction de l'âge du patient : on peut considérer que chez un « jeune sénior » ( $<75$ ans) qui chute ou souffre d'une altération importante de la marche, les seuls effets du vieillissement ne peuvent expliquer la situation jusqu'à preuve du contraire. Dans cette tranche d'âge, le recours aux examens complémentaires ou à des avis spécialisés sera donc beaucoup plus large. Chez les sujets très âgés (85 ans et plus) au contraire certaines constatations n'impliquent pas l'existence d'une pathologie et sont attribuables au vieillissement, dans cette population et chez des personnes en bonne santé par ailleurs, la manœuvre de Romberg est positive dans $47 \%$ des cas, les réflexes achilléens abolis chez $56 \%$ des sujets, la sensibilité vibratoire et sens de position des orteils diminués respectivement chez 67 et $56 \%$ des sujets [4]. De telles constatations ne doivent donc pas déclencher automatiquement des explorations.

Quelques points particuliers nous semblent importants à souligner. Dans le contexte d'un syndrome parkinsonien, il faut garder à l'esprit que les chutes ne sont pas un signe de début de la maladie de Parkinson idiopathique, et n'apparaissent que tardivement dans l'évolution. Au contraire les chutes sont le principal signe d'appel de la paralysie supranucléaire progressive (maladie de Steele Richardson), alors même que la marche reste de bonne qualité, de même dans le contexte d'un syndrome multilacunaire qui peut être pauci-symptomatique. Lorsqu'il existe une astasie abasie c'est-à-dire une impossibilité complète ou quasi-complète à se mettre debout et marcher, avec abolition des réflexes de posture, que l'on peut assimiler à « l'apraxie de la marche » [26], une imagerie cérébrale est indispensable, même s'il n'y a pas d'autre signe d'examen, à la recherche d'une lésion sous-corticofrontale tumorale ou vasculaire, d'un hématome sous-dural ou encore d'une hydrocéphalie aiguë ou chronique [26].

L'étape suivante consiste à évaluer le risque de récidive de chute, un grand nombre de tests et d'échelles plus ou moins complexes ont été proposés (pour une revue voir : référentiel concernant I'évaluation du risque de chutes et sa prévention chez le sujet âgé autonome. HAS 2012, www.has- 
sante.fr). En pratique quelques épreuves simples et rapides donnent les meilleures indications, dans notre expérience il s'agit :

- Du « timed up and go test » : épreuve chronométrée où le patient doit se relever d'un siège avec accoudoir, marcher trois mètres, faire demi-tour et se rasseoir. Un temps supérieur à 14 secondes est prédictif d'un risque de chute.

- De la manœuvre de Romberg

- De la station monopodale, qui, si elle ne peut être maintenue plus de 5 secondes est fortement prédictive du risque de chute.

- De l'épreuve de la poussée sternale, l'examinateur se place derrière le patient et applique une poussée sternale modérée. L'altération des réflexes d'adaptation posturale se traduit par la nécessité de plusieurs pas en arrière pour se stabiliser ou, au maximum, par l'absence de réaction avec nécessité de l'intervention de l'examinateur.

Il faut cependant noter que la sensibilité de ces épreuves n'atteint qu'environ $40 \%$, c'est plutôt la valeur prédictive négative qui est élevée, de l’ordre de $95 \%$ : si toutes les épreuves sont réussies, le risque de chute est très faible.

Les données anamnestiques donnent aussi de précieuses indications pronostiques, notamment un antécédent de chute et l'impossibilité de se relever seul, deux éléments non seulement associés aux récidives mais aussi au risque d'institutionnalisation et de décès à 12 mois $[15,23]$.

\section{La prise en charge}

Au terme de l'évaluation clinique globale un certain nombre de questions doivent avoir reçu une réponse permettant de guider la conduite à tenir.

Sur les circonstances de la chute : chute fortuite (accidentelle) liée à un élément extérieur qui aurait pu faire chuter tout un chacun, dans ce cas il n'est pas obligatoire de proposer une intervention s'il n'y a pas de syndrome post-chute. Chute dans un contexte pathologique spécifique aigu (par exemple syndrome fébrile) ou chronique (par exemple hypoglycémie chez un diabétique), qui devra déboucher sur des traitements adaptés à chaque cas. Mais le plus souvent chez les sujets âgés troubles de la marche et chutes sont polyfactoriels et les différents déterminants ne sont pas toujours accessibles à une thérapeutique, à commencer par l'âge lui-même... 
D’une façon générale, il faut s'attacher à éliminer les facteurs favorisants.

Les facteurs de l'environnement (dit « extrinsèques »), au mieux avec l'aide d'un ergothérapeute ou d'un autre intervenant à domicile qui pourra préconiser des mesures de bon sens (pourtant pas toujours bien acceptées [25]) telles que supprimer les tapis et les meubles embarrassant les couloirs, porter des chaussures adaptées (fermées et à semelle fine), veiller à un éclairage suffisant avec éventuellement veilleuses ou détecteurs de mouvement, installer des barres de soutien dans les toilettes et la salle de bain... L'éducation du patient et de son entourage par un professionnel de santé sur les activités à risque et les précautions à prendre pour minimiser les récidives. Ces simples mesures pourraient réduire le nombre de chutes de 20 \% [25], mais l'évaluation précise de leur efficacité est difficile. Chez le sujet chuteur ou à risque de chute, il faut aussi proposer l'installation d'une téléalarme (ou téléassistance) : à partir d'un boîtier porté en permanence la personne peut alerter un téléopérateur qui déclenchera, le cas échéant, l'intervention des secours ; en cas de non réponse au rappel téléphonique par exemple. Il s'agit cependant d'un service payant (20 à 30 euros par mois en moyenne).

Les facteurs propres à l'individu (dit « intrinsèques »), comprennent les pathologies accessibles à un traitement (chirurgie de la cataracte, prothèse de hanche, ajustement d'un traitement antiparkinsonien, traitement d'un trouble du rythme ou de la conduction cardiaque...). Réviser l'ordonnance du patient fait aussi partie des priorités en éliminant autant que possible les psychotropes et les médicaments susceptibles d'entraîner une hypotension orthostatique.

Le dernier volet de la prise en charge concerne les interventions spécifiques en vue de prévenir chutes et fractures, il est conseillé d'introduire une supplémentation en calcium et vitamine $D$, bien que les preuves d'efficacité sur le risque de chute et fracturaire soient discutées [27]. La plupart des interventions préconisées font appel à la physiothérapie axée sur des exercices de marche et d'équilibration, un travail d'assouplissement articulaire notamment aux membres inférieurs, un travail de renforcement musculaire, adapté à chacun et visant à renforcer la ceinture pelvienne et les triceps suraux [5]. D’autres activités spécifiques peuvent être ciblées selon les cas : exercices sur les transferts, entraînement à se remettre debout depuis la position allongée sur le sol sur le ventre et sur le dos. Le cas échéant, le kinésithérapeute devra superviser la mise en place d'aides techniques : canne, déambulateur voire fauteuil roulant qu'il ne faut pas hésiter à proposer et qui doit être vu comme un instrument d'autonomisation et présenté comme tel à des sujets souvent réticents... II existe aussi des protecteurs de hanche, dont l'efficacité est discutée et dont l'acceptabilité est médiocre du fait de l'inconfort engendré par le dispositif. 
L'ensemble de cette prise en charge incluant la correction des facteurs favorisants et la physiothérapie donne cependant des résultats décevants sur la réduction du risque de chute. Une récente métaanalyse de la Cochrane Collaboration [28] qui a analysé 62 études, conclue à des preuves d'efficacité faibles à très faibles... Seul le nombre total de chutes par année semble réduit : 1784 pour 1000 personnes dans le groupe intervention contre 2317 dans le groupe recevant des soins usuels. Mais il n'y a pas de différence significative pour ce qui est du nombre de chuteurs, du nombre d'hospitalisations pour chute ni du nombre de fractures. Une autre méta-analyse conclue à une efficacité modérée de ces mêmes interventions pour améliorer le « syndrome de peur de la chute » (avec les problèmes de définition de cette entité déjà mentionnés) [29]. Ces résultats négatifs dans l'ensemble ne doivent cependant pas faire renoncer à ce type de prise en charge, il nous semble probable que chez des sous-groupes de patients elle a une utilité, notamment si l'on concentre l'intervention sur les sujets indemnes de troubles cognitifs [18]. De même lorsque l'on a à faire à un syndrome post-chute aigu, une prise en charge rapide avec verticalisation et reprise d'activité de la vie quotidienne est certainement efficace, a contrario plus l'alitement se prolonge, plus le risque de perte d'autonomie est grand [15].

\section{Conclusions}

Avec le vieillissement global de la population, troubles locomoteurs et chutes deviennent un problème majeur de santé publique tant par la morbi-mortalité qu'ils engendrent que par les ressources humaines et financières qui doivent y être consacrées. Jusqu'à présent les interventions a posteriori n’ont donné que des résultats décevants. Aussi faudrait-il envisager une démarche de prévention primaire lorsque les sujets ont encore leur autonomie fonctionnelle, même si une étude prospective à grande échelle reste à faire ; par des conseils d'hygiène de vie, en soulignant notamment l'importance d'une activité physique modérée quotidienne, par la prévention, quand elle est possible, ou tout au moins la prise en charge précoce des affections chroniques qu'elles soient ophtalmologiques, rhumatologiques, neurodégénératives ou encore cardio-vasculaires qui doivent être dépistées.

Déclaration d'intérêts : l'auteur ne déclare aucun conflit d'intérêt. 


\section{Références}

[1] Bergen G, Stevens MR, Burns ER. Falls and falls injuries among adults aged $\geq 65$ years - United States, 2014. MMWR Morb Mortal Wkly Rep 2016; 65: 993-998.

[2] Tinetti ME, Speechley M, Ginter SF. Risk factors for falls among elderly persons living in the community. N Engl J Med 1988; 319: 1701-7.

[3] Rothwell J. Control of human voluntary movement, second edition 1994. Chapman \& Hall. London.

[4] Kaye JA, Oken OS, Howieson DB et al. Neurologic evaluation of the optimally healthy oldest old. Arch Neurol 1994; 51: 1205-11.

[5] Sturnieks DL, St George R, Lord SR. Balance disorders in the elderly. Neurophysiol Clin 2008; 38: 467-78.

[6] Bergström B. Morphology of the vestibular nerve. III. Analysis of the calibers of the myelinated vestibular nerve fibers in man at various ages. Acta Otolaryngol 1973; 76: 331-338.

[7] Agmon M, Lavie L, Doumas M. The association between hearing loss, postural control, and mobility in older adults : a systematic review. J Am Acad Audiol 2017; 28: 575-88.

[8] Stelmach GE, Worringham CJ. Sensorimotor deficits related to postural stability. Implications for falling in the elderly. Clinics in Geriatric Medicine 1985; 1: 679-94.

[9] Foldvari M, Clark M, Laviolette LC et al. Association of muscle power with functional status in community-dwelling elderly women. J Gerontol A Biol Sci Med Sci 2000; 55: 192-99.

[10] Herman T, Mirelman A, Giladi N, et al. Executive control deficits as a prodrome to falls in healthy older adults : a prospective study linking thinking, walking, and falling. J Gerontol A Biol Sci Med Sci $2010 ; 65: 1086-92$.

[11] Li KZH, Bherer L, Mirelman A, et al. Cognitive involvement in balance, gait and dual-tasking in aging : a focused review from a neuroscience of aging perspective. Front Neurol 2018; 9: 913. eCollection 2018.

[12] Lundin-olsson L, nyberg L, gustafson Y. 《Stops walking when talking 》 as a predictor of falls in elderly people. Lancet 1997; 349: 617. 
[13] Nutt JG, Marsden CD, Thomson PD. Human walking and higher-level gait disorders, particularly in the elderly. Neurology 1993; 43: 268-79.

[14] Maki BE. Gait changes in older adults : predictors of falls or indicators of fear. J Am Geriatr Soc 1997; 45: 313-20.

[15] Murphy J, Isaacs B. The post-fall syndrome. A study of 36 elderly patients. Gerontology 1982; 28: $265-70$.

[16] Scheffer AC, Schuurmans MJ, Van Dijk N et al. Fear of falling : measurement strategy, prevalence, risk factors and consequences among older persons. Age and Ageing 2008; 37: 19-24.

[17] Makino K, Makizako H, Doi T et al. Fear of falling and gait parameters in older adults with and without fall history. Geriatr Gerontol Int 2017; 17: 2455-9.

[18] Kueper JK, Speechley M, Lingum NR et al. Motor function and incident dementia : a systematic review and meta-analysis. Age and Ageing 2017; 46: 729-38.

[19] Deandrea S, Lucenteforte E, Bravi F, et al. Risk factors for falls in community-dwelling older people : a systematic review and meta-analysis. Epidemiology 2010; 21: 658-68.

[20] Woolcot JC, Richardson KJ, Wiens MO, et al. Meta-analysis of the impact of 9 medication classes on falls in elderly persons. Arch Intern Med 2009; 169: 1952-60.

[21] Allain $H$, Bentué-ferrer $D$, Polard E et al. Postural instability and consequent falls and hip fractures associated with use of hypnotics in the elderly : a comparative review. [22] Ermanel C, Thélot B, Jougla E, et al. Mortalité par accident de la vie courante en France métropolitaine, 2000-2004. Bulletin Epidémiologique Hebdomadaire 2007. Numéro thématique ; 37-38 : 318-22.

[23] Tinetti ME, Williams CS. Falls, injury due to falls, and the risk of admission to a nursing home. N Engl J Med 1997; 337: 1279-84.

[24] Burns ER, Stevens JA, Lee R. The direct costs of fatal and non-fatal falls among older adultsUnited States. J Safety Res 2016; 58: 99-103.

[25] Tinetti ME. Preventing falls in elderly persons. N Engl J Med 2003; 348: 42-49.

[26] Dale ML, Curtze C, Nutt JG. Apraxia of gait- or apraxia of postural transitions? Parkinsonism and related disorders 2018; 50: 19-22. 
[27] Zhao JG, Zeng XT, Wang J, et al. Association between calcium or vitamin D supplementation and fracture incidence in community-dwelling older adults : a systematic review and meta-analysis. JAMA 2017; 318: 2466-82.

[28] Hopewell S, Adedire O, Copsey BJ, et al. Multifactorial and multiple component interventions for preventing falls in older people living in the community. Cochrane Database of Systematic Reviews 2018: CD012221. DOI : 10.1002/14651858.CD012221.pub2.

[29] Kendrick D, Kumar A, Carpenter H D, et al. Exercise for reducing fear of falling in older people living in the community. Cochrane Database of Systematic Reviews 2014. : CD009848.DOI :

10.1002/14651858.CD009848.pub2.tabke 